EPJ Web of Conferences 101, 04007 (2015)

DOI: $10.1051 /$ epjconf/ 201510104007

(C) Owned by the authors, published by EDP Sciences, 2015

\title{
Heartbeat Stars and The Ringing of Tidal Pulsations
}

\author{
Kelly Hambleton ${ }^{1,2, a}$, Don Kurtz ${ }^{1}$, Andrej Prša ${ }^{2}$, Jim Fuller ${ }^{3}$, and Susan Thompson ${ }^{4,5}$ \\ 1 Jeremiah Horrocks Institute, University of Central Lancashire, Fylde Road, Preston, Lancashire, \\ PR1 2HE England \\ 2 Department of Astrophysics and Planetary Science, Villanova University, 800 East Lancaster Av- \\ enue, Villanova, PA 19085 USA \\ 3 California Institute of Technology, 1200 E California Blvd, Pasadena, CA 91125 USA \\ 4 NASA Ames Research Center, Moffett Field, CA 94035, USA \\ 5 SETI Institute, 189 Bernardo Avenue, Suite 100, Mountain View, CA 94043, USA.
}

\begin{abstract}
With the advent of high precision photometry from satellites such as Kepler and CoRoT, a whole new layer of interesting and astounding astronomical objects has been revealed: heartbeat stars are an example of such objects. Heartbeat stars are eccentric ellipsoidal variables that undergo strong tidal interactions when the stars are almost in contact at the time of closest approach. These interactions deform of the stars and cause a notable light curve variation in the form of a tidal pulse. A subset of these objects $(\sim 20 \%)$ show prominent tidally induced pulsations: pulsations forced by the binary orbit. We now have a fully functional code that models binary star features (using PHOEBE) and stellar pulsations simultaneously, enabling a complete and accurate heartbeat star model to be determined. In this paper we show the results of our new code, which uses EMcEe, a variant of MCMc, to generate a full set of stellar parameters. We further highlight the interesting features of KIC 8164262, including its tidally induced pulsations and resonantly locked pulsations.
\end{abstract}

\section{Introduction}

In close binary systems, the tidal interactions between the stellar components cause the stars to become distorted and prolate, with the point of the star extending towards the companion. Consequently, as the stars progress around the orbit and the stellar profile and temperature distribution over the star changes, we observe variations in the stellar flux. When combined with luminosity variations produced by reflection and Doppler boosting, this phenomenon is known as ellipsoidal variation. In circular systems the light varies periodically, twice per orbit. In eccentric systems, such as heartbeat stars, the same physical principles apply, although due to the varying distance between the stars over the orbit, the stellar shapes are also constantly changing, with the most significant change occurring when the stars are at closest approach. Thus, in these systems, the dominant variation in the light curve (known as the periastron variation or heartbeat) occurs once per orbital cycle at periastron.

The first heartbeat star, KOI-54 (depicted in the top panel of Fig. 1), was discovered using the Kepler satellite (1). Shortly after, many more discoveries were made including those by Thompson et al. (2), who published a catalogue of 17 heartbeat stars from the Kepler field; Nicholls \& Wood (3), who identified seven heartbeat stars in the Large Magellanic Cloud using the OGLE survey; and more recently in the Kepler binary catalogue 3 paper (Kirk et al. 2014, in prep.), an extensive catalogue of binary stars that contains 154 heartbeat stars, all observed using the Kepler satellite. Fig. 1 shows a selection of heartbeat stars from the Kepler sample. These heartbeat stars were identified through

\footnotetext{
a e-mail: kmhambleton@uclan.ac.uk
}

This is an Open Access article distributed under the terms of the Creative Commons Attribution License 4.0, which permits unrestricted use, distribution, and reproduction in any medium, provided the original work is properly cited. 


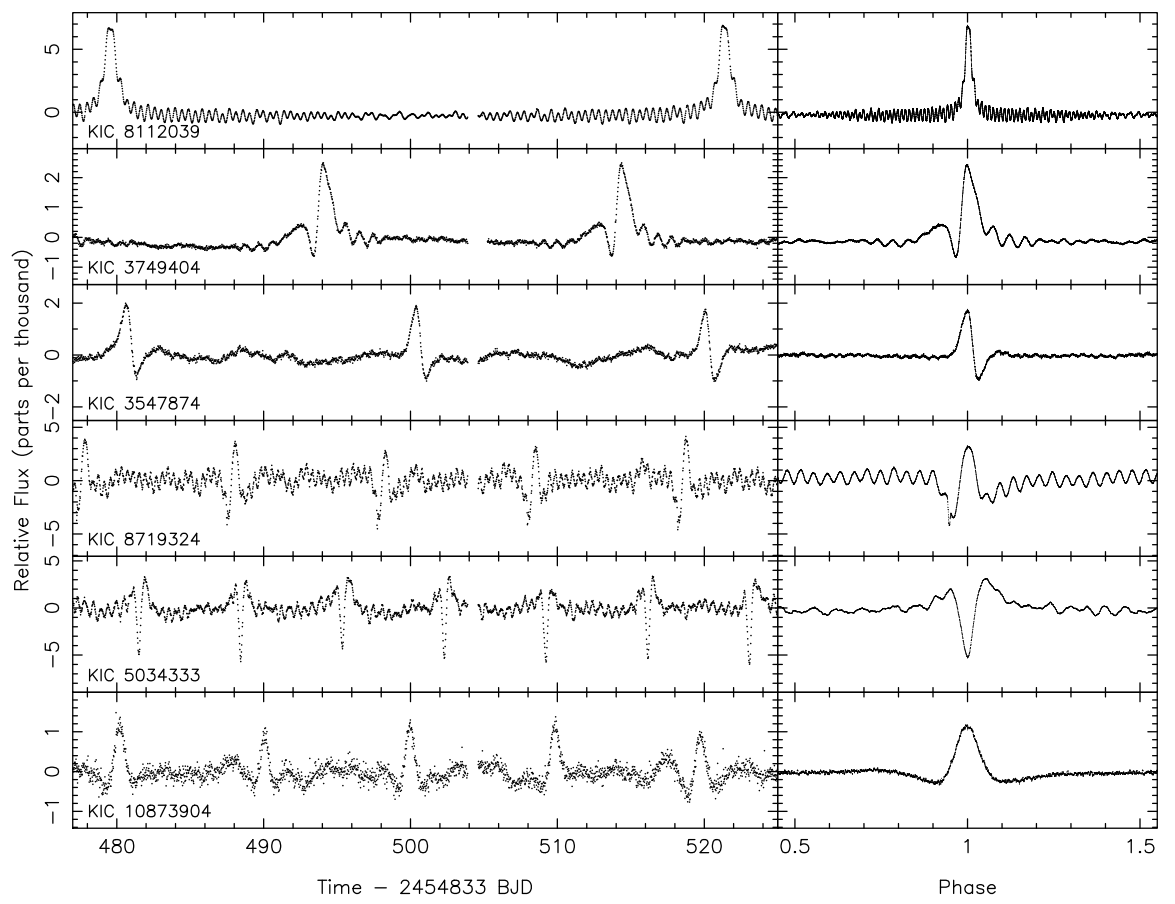

Fig. 1. A selection of heartbeat stars from the Kepler sample. Depicted are the time series (left panel) and phase folded data (right panel). The phase folded data clearly depict the pulsations that are integer multiples of the orbital frequency: tidally induced pulsations.

a combined effort from the Heartbeat Star Team, the Planet Hunters, the Eclipsing Binary Working Group and other independent sources.

\section{Tidally Induced Pulsations}

Tidally induced pulsations are stellar oscillations that are driven by the tidal force of the companion star. In a binary system with an eccentric orbit, when a stellar eigenfrequency is close to a multiple of the orbital frequency, the star can act like a forced oscillator, which causes an increase in the oscillation amplitude of the mode (relative to non-resonant modes). The signature of tidally excited modes is thus an oscillation frequency at a precise integer multiple of the orbital frequency. Approximately $20 \%$ of our current Kepler sample of heartbeat stars demonstrate tidally induced pulsations above our detection limit.

Tidally induced pulsations have long been theorised $(4 ; 5 ; 6)$ as a mechanism for the circularisation of binary star orbits and the spin up of stars. However, until KOI-54, their presence has only been speculated (7). The unquestionable nature of the tidally induced pulsations in KOI-54 sparked the interest of many theorists and consequently three publications $(8 ; 9 ; 10)$ appeared discussing the physical nature of tidally induced pulsations and the relevance of the $90^{\text {th }}$ and $91^{\text {st }}$ orbital harmonic - the two prominent pulsation frequencies in KOI-54. Fuller \& Lai (8) proposed that the two highest amplitude tidally induced pulsations were excited through a mechanism called resonant locking (cf. §2.1). Burkart et al. (9) disputed this on the grounds that the torque produced by the proposed modes was not enough to provide resonant locks and that it is unlikely that two resonant locks would occur simultaneously. Later, O'leary \& Burkart (10) showed that the $90^{\text {th }}$ and $91^{\text {st }}$ harmonic were likely $l=2, m=0$ modes, which theory at the time suggested could not be resonantly locked. Another interesting observation by O'leary \& Burkart (10) was that all of the pulsation modes of KOI-54 are 
either tidally excited pulsations or their combinations, which was later found to be the case for all the gravity (g) modes in KIC 4544587 (11).

\subsection{Resonant Locking}

The theory of resonantly excited modes - eigenmodes in resonance with the orbit - was suggested by Witte and Savonije $(12 ; 13)$ and again more recently by Kumar, Ao and Quataert (14) long before the discovery of heartbeat stars. Due to the expected transient nature of resonant modes, it was not anticipated that they would be observed. However, KOI-54 and KIC 8164262 (cf. Sect. 4) both have high amplitude modes that are not easily explained by the forcing of the eigenfrequencies alone. Resonance locking has been proposed as a mechanism that can "lock" a stellar eigenfrequency and the orbit in resonance, thus extending the lifetime of the resonance and making observations of tidal resonance possible. While the resonant locking of KOI-54 is currently under dispute, it has not been completely ruled out. Moreover, KIC 8164262 has only a single high amplitude mode that is an $l=2, m=2$ mode, which makes a stronger case for resonant locking.

The proposed mechanism of resonant locking is the following: as two stars in an eccentric configuration orbit each other, the stellar orbit evolves through the transfer of energy and angular momentum, causing the orbit and thus orbital period to get smaller and the stars spin faster. In parallel, the increase in rotational velocity of the stars causes the stellar eigenfrequencies to change. Resonance locking predicts that, when in resonance, these two effects happen on an equal time scale such that the stellar eigenfrequencies are locked to the changing orbital period. Thus, rather than passing through resonance the tidally induced pulsations are locked in resonance with the orbit, enabling the observation of resonantly excited modes.

\section{Modelling Heartbeat Stars}

As the parameter space of binary stars is highly irregular with many degeneracies and correlations, we chose to combine EMCEE, an affine invariant version of Markov chain Monte Carlo methods (MCMC) (15) with PHOEBE, the binary modelling software package, which now has PYTHON scripting capabilities. When modelling heartbeat stars we also fit the tidally induced pulsations using sine waves by fixing the frequencies to a multiples of the orbital frequency and fitting the amplitudes and phases.

To reduce the number of parameters in our models, at each iteration we calculate: the phase of the periastron variation, the passband luminosity and the spin to orbital rotation frequency. To enhance our models we also calculate the critical potentials at the Lagrangian points $L_{1}$ and $L_{2}$. Subsequently, if there is no evidence of mass transfer, we use this as a constraint for our models and do not allow the potential value to become smaller than the potential at the Lagrangian point $L_{1}$. Alternatively, if there is evidence of mass transfer (or for contact systems), we do not allow the stellar potentials to become smaller than $L_{2}$ as this creates a non-physical scenario where the model star has a dimple in the side pointing away from the companion. The common geometry of heartbeat stars also make them ideal candidates for Doppler boosting. Consequently we adjust the model flux to account for Doppler boosting using Eqn. 2 in Bloemen et al. (16), extended to account for boosting in both objects.

\section{KIC 8164262}

KIC 8164262 is a highly eccentric $(e \approx 0.9)$, 13th magnitude heartbeat star in a $\sim 90 \mathrm{~d}$ orbit with a single high amplitude $(\sim 900 \mathrm{ppm})$ tidally induced pulsation that oscillates precisely 229 times per orbit (cf. Fig.2). The primary component of KIC 8164262 is a late A/early F star with an M type companion. This object was selected for detailed study as the prominent high amplitude mode was suggestive of resonant locking. KIC 8164262 presents a plethora of tidally induced g modes ranging from 120 to 320 cycles per orbit $\left(\sim 1-4 \mathrm{~d}^{-1}\right)$. However, the largest amplitude mode has an amplitude twenty times greater than all other modes ( $900 \mathrm{ppm}$ compared to the second highest at $45 \mathrm{ppm})$. We 


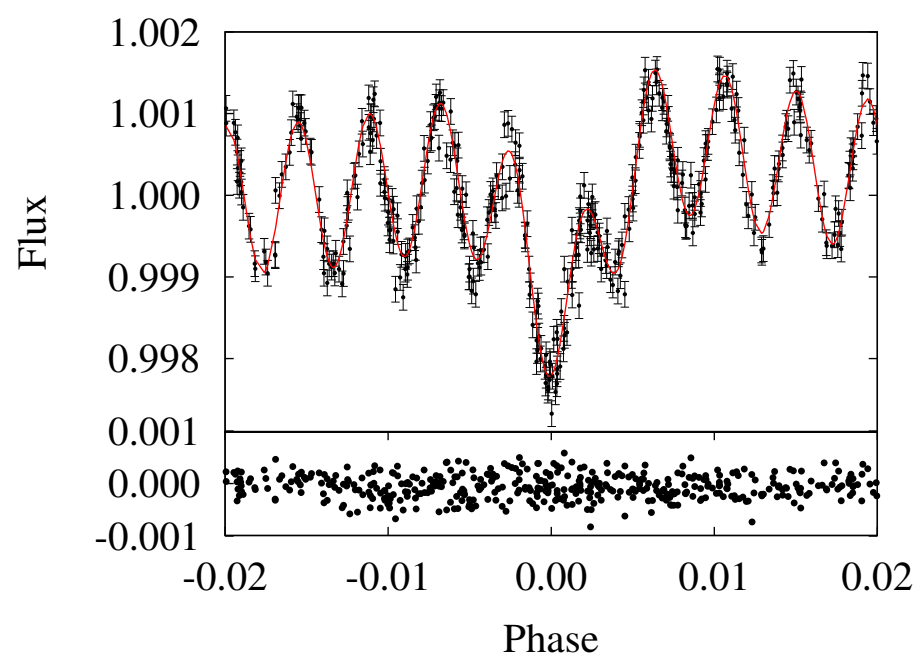

Fig. 2. Upper panel: A phased light curve (black points) of KIC 8164262 , a heartbeat star with a single high amplitude pulsation. The model is overlayed in red and is comprised of the binary features and highest amplitude pulsation. The periastron variation, centred on zero, has a similar amplitude to the highest amplitude pulsation. Lower panel: the residuals of the binary model fit to the phased data (black points). Only a fraction of the $90 \mathrm{~d}$ orbit is shown for clarity.

used theoretical models of a star with equal mass $\left(M_{1}=1.9 \mathrm{M}_{\odot}\right)$, radius $\left(R_{1}=2.5 \mathrm{R}_{\odot}\right)$ and temperature $\left(\mathrm{T}_{e f f, 1}=7000 \mathrm{~K}\right)$ to predict the expected combinations of frequency and amplitudes for resonantly locked modes. These predictions are in good agreement with the frequency and amplitude of the $229^{\text {th }}$ harmonic.

\section{References}

1. W.F. Welsh, J.A. Orosz, C. Aerts, T.M. Brown, E. Brugamyer, W.D. Cochran, R.L. Gilliland, J.A. Guzik, D.W. Kurtz, D.W. Latham et al., ApJS197, 4 (2011), 1102 . 1730

2. S.E. Thompson, M. Everett, F. Mullally, T. Barclay, S.B. Howell, M. Still, J. Rowe, J.L. Christiansen, D.W. Kurtz, K. Hambleton et al., ApJ753, 86 (2012), 1203.6115

3. C.P. Nicholls, P.R. Wood, MNRAS421, 2616 (2012), 1201.1043

4. J.P. Zahn, A\&A41, 329 (1975)

5. P. Goldreich, P.D. Nicholson, ApJ342, 1079 (1989)

6. M.G. Witte, G.J. Savonije, A\&A386, 222 (2002)

7. C. Maceroni, J. Montalbán, E. Michel, P. Harmanec, A. Prsa, M. Briquet, E. Niemczura, T. Morel, D. Ladjal, M. Auvergne et al., A\&A508, 1375 (2009), 0910. 3513

8. J. Fuller, D. Lai, MNRAS420, 3126 (2012), 1107 . 4594

9. J. Burkart, E. Quataert, P. Arras, N.N. Weinberg, MNRAS421, 983 (2012), 1108 . 3822

10. R.M. O’Leary, J. Burkart, MNRAS440, 3036 (2014), 1308.0016

11. K.M. Hambleton, D.W. Kurtz, A. Prša, J.A. Guzik, K. Pavlovski, S. Bloemen, J. Southworth, K. Conroy, S.P. Littlefair, J. Fuller, MNRAS434, 925 (2013), 1306. 1819

12. M.G. Witte, G.J. Savonije, A\&A350, 129 (1999), astro-ph/9909073

13. M.G. Witte, G.J. Savonije, A\&A366, 840 (2001)

14. P. Kumar, C.O. Ao, E.J. Quataert, ApJ449, 294 (1995), arXiv:astro-ph/9503053

15. D. Foreman-Mackey, D.W. Hogg, D. Lang, J. Goodman, PASP125, 306 (2013), 1202 . 3665

16. S. Bloemen, T.R. Marsh, R.H. Østensen, S. Charpinet, G. Fontaine, P. Degroote, U. Heber, S.D. Kawaler, C. Aerts, E.M. Green et al., MNRAS410, 1787 (2011), 1010.2747 